OPEN ACCESS

Edited by:

Guilhian Leipnitz,

Federal University of Rio Grande do

Sul, Brazil

Reviewed by:

Dean Appling,

University of Texas at Austin,

United States

Nelson L. S. Tang,

The Chinese University of Hong Kong,

China

*Correspondence:

Nicholas D. E. Greene

n.greene@ucl.ac.uk

orcid.org/0000-0002-4170-5248

Specialty section:

This article was submitted to Genetics of Common and Rare

Diseases,

a section of the journal

Frontiers in Genetics

Received: 02 November 2020 Accepted: 04 January 2021

Published: 25 January 2021

Citation:

Leung K-Y, De Castro SCP,

Galea GL, Copp AJ and Greene NDE (2021) Glycine Cleavage System H

Protein Is Essential for Embryonic Viability, Implying Additional Function Beyond the Glycine Cleavage System.

Front. Genet. 12:625120.

doi: 10.3389/fgene.2021.625120

\section{Glycine Cleavage System H Protein Is Essential for Embryonic Viability, Implying Additional Function Beyond the Glycine Cleavage System}

\author{
Kit-Yi Leung, Sandra C. P. De Castro, Gabriel L. Galea, Andrew J. Copp and \\ Nicholas D. E. Greene*t
}

Great Ormond Street Institute of Child Health, University College London, London, United Kingdom

Glycine cleavage system $\mathrm{H}$ protein $(\mathrm{GCSH})$ is a component of the glycine cleavage system (GCS), a conserved protein complex that acts to decarboxylate glycine. Mutation of $A M T$ or GLDC, encoding the GCS components aminomethyltransferase and glycine decarboxylase, can cause malformations of the developing CNS (neural tube defects (NTDs) and ventriculomegaly) as well as a post-natal life-limiting neurometabolic disorder, Non-Ketotic Hyperglycinemia. In contrast, it is unclear whether mutation of GCSH contributes to these conditions and we therefore investigated GCSH loss of function in mice. Mice that were heterozygous for a Gcsh null allele were viable and did not exhibit elevated plasma glycine. Moreover, heterozygous mutation of Gcsh did not increase the frequency of NTDs in Gldc mutant embryos. Homozygous Gcsh null mice were not recovered at post-natal stages. Analysis of litters at E8.5-10.5, revealed the presence of homozygous null embryos which were much smaller than littermates and had failed to develop beyond early post-implantation stages with no visible somites or head-folds. Hence, unlike null mutations of Gldc or Amt, which are compatible with embryonic survival despite the presence of NTDs, loss of Gcsh causes embryonic death prior to mid-gestation. Maternal supplementation with formate did not restore embryonic development beyond $\mathrm{E7.5}$, suggesting that the primary cause of lethality was not loss of glycine cleavage activity or suppression of folate one-carbon metabolism. These findings suggest that GCSH has additional roles beyond function in the glycine cleavage system. We hypothesize that GCSH potentially acts in lipoylation of 2-oxoacid dehydrogenase proteins, as reported in bacteria.

Keywords: glycine cleavage system, glycine cleavage system H protein, embryonic lethality, lipoylation, mouse models

\section{INTRODUCTION}

Glycine cleavage system H-protein (GCSH) is one of four enzymes which, together with glycine decarboxylase (GLDC), aminomethyltransferase (AMT), and dehydrolipamide dehydrogenase (DLD), make up the glycine cleavage system (GCS). This highly conserved protein complex is located at the mitochondrial membrane in eukaryotes and is the major route of glycine catabolism. 
GCS action involves oxidative cleavage of glycine with release of carbon dioxide $\left(\mathrm{CO}_{2}\right)$ and ammonia $\left(\mathrm{NH}_{3}\right)$ and transfer of a methylene group $\left(-\mathrm{CH}_{2}-\right)$ to tetrahydrofolate, with concomitant reduction of $\mathrm{NAD}^{+}$to NADH (Figure 1A; Kikuchi et al., 2008).

Based on analysis in the leaf of pea plants (Pisum sativum), the stoichiometry of the GCS is estimated at 4 GLDC :27 GCSH: 9 AMT :2 DLD (Oliver et al., 1990). GCSH plays a central role in the catalytic process, forming an amino-methyl intermediate with GLDC and acting as the acceptor of the methylamine group which is then transferred as the substrate for AMT (Figure 1A). This function of GCSH depends on modification by covalent addition of lipoic acid, as a cofactor, to form the active lipoylenzyme. The lipoyl moiety is believed to assembled on GCSH in a multi-step process, with addition of the octanoyl group followed by insertion of the sulphydryl groups (Figure 1B; Christensen et al., 2011; Cao et al., 2018b; Cronan, 2020). DLD mediates reduction of $\mathrm{NAD}^{+}$and oxidation of the lipoyl group of GCSH (Figure 1A).

Loss of function of the GCS is predicted to cause accumulation of glycine and suppression of folate one-carbon metabolism (FOCM) and this is associated with post-natal neurometabolic disease and structural malformations of the developing brain including neural tube defects (NTDs) and ventriculomegaly. Accumulation of glycine in tissue and body fluids is a hallmark of Non-Ketotic Hyperglycinemia (NKH), a life-limiting inborn error of metabolism which presents in the neonatal period with hypotonia, apnea, and seizures. Subsequently, affected babies and children suffer complex epilepsy and profound developmental delay (Hoover-Fong et al., 2004; Swanson et al., 2015). Enlarged brain ventricles and/or hydrocephalus are also associated with $\mathrm{NKH}$ in a proportion of patients (Van Hove et al., 2000; Hennermann et al., 2012). Classic NKH, caused by mutation of GCS-encoding genes, is inherited as an autosomal recessive condition with the majority of patients carrying mutations in GLDC and around 20\% carrying mutations in AMT (Kure et al., 2006; Kanno et al., 2007; Coughlin et al., 2017).

In addition to $\mathrm{NKH}$, mutations in GLDC and AMT contribute to susceptibility to NTDs (Narisawa et al., 2012; Shah et al., 2016), severe birth defects which result from failed closure of the neural tube during embryonic development. To date, GCS mutations reported in NTDs are in heterozygous form suggesting that mutations in other genes and/or environmental factors also contribute to these cases, consistent with the multifactorial etiology of NTDs (Greene and Copp, 2014).

The potential role of GLDC and AMT mutations in some NTDs is supported by the occurrence of NTDs in mouse knockouts of the orthologous genes (Narisawa et al., 2012; Pai et al., 2015; Leung et al., 2017). Among Gldc-deficient mice that are not affected by NTDs, the occurrence of ventriculomegaly and hydrocephalus (resulting from stenosis of the aqueduct of Sylvius) similarly confirm that these are specific effects of GCS-encoding mutations in NKH patients (Autuori et al., 2017; Santos et al., 2020).

The loss of GCS activity in Gldc-deficient mice results in elevated glycine and a number of glycine derivatives in plasma and tissues, including liver and brain (Pai et al., 2015; Leung et al., 2020). In parallel, metabolic labeling shows that the contribution of glycine-derived one-carbon units to folate onecarbon metabolism is ablated (Leung et al., 2017) and the folate profile is perturbed, with a deficit of folates that carry one-carbon groups (Pai et al., 2015).

Whereas DLD acts in at least three other mitochondrial enzyme complexes, GLDC, AMT, and GCSH have been described as specific to the glycine cleavage system. However, unlike GLDC and $A M T$, mutations in GCSH have not been reported in patients with NTDs or NKH (Kure et al., 2006; Coughlin et al., 2017). However, a putative heterozygous mutation was identified in an individual with transient neonatal hyperglycinemia (Kure et al., 2002). In addition, patients have been identified in which elevated plasma glycine is associated with reduced GCSH activity and defects in addition of the lipoic acid moiety (Hiraga et al., 1981), with mutations identified in lipoic acid synthase, LIAS (Baker et al., 2014). In the current study, we investigated the effect of Gcsh loss of function in mice, in order to ask whether this revealed a potential role in NTDs and/or features of $\mathrm{NKH}$.

\section{MATERIALS AND METHODS}

\section{Mice}

Animal studies were carried out under regulations of the Animals (Scientific Procedures) Act 1986 of the United Kingdom Government, and in accordance with the guidance issued by the Medical Research Council, United Kingdom in Responsibility in the Use of Animals for Medical Research (July 1993). The Gcsh ${ }^{\mathrm{em} 1(\mathrm{IMPC}) \mathrm{H}}$ (previously denoted H-GCSH-DEL653-EM1B6N) mouse line was generated by CRISPR/Cas9 targeting at MRC Harwell, Mary Lyon Centre (Oxfordshire) on a C57BL/6N Tac background (Mianne et al., 2017). The Gcsh mutant allele (hereafter denoted $\mathrm{Gcsh}^{-}$) carried a deletion of 653 nucleotides (nucleotides 116,987,776-116,987,124) encompassing exon 3 of Gcsh (ENSMUSG00000034424).

\section{Genotyping}

Genotyping of mice and embryos was carried out by PCR amplification of genomic DNA. Use of primers which flank the deletion (Gcsh_F2;5'-GCACGTAGTAGGGTTGACAAGT$3^{\prime}$ and Gcsh_R2; 5'-CGCGGATGGAGAACTGTAAGG-3') produces a PCR product of $1,033 \mathrm{bp}$ from wild-type DNA whereas the deletion allele generates a smaller product of 380 bp. A confirmatory reaction (Gcsh_F2 primer and Gsch_R1; 5' CAGGAAGCGTTGGGAGATGTTG-3') used a reverse primer which binds in the deleted region and therefore generated PCR product from wild-type but not Gcsh deleted DNA.

\section{Generation of $\mathrm{Gldc}^{-/-} ; \mathrm{Gcsh}^{+/-}$Mice}

Gldc-deficient mice, denoted Gldc ${ }^{\mathrm{GT} 1}$, carry a gene-trap construct in intron 2 of Gldc (Pai et al., 2015) and are maintained on a $\mathrm{C} 57 \mathrm{Bl} / 6 \mathrm{~J}$ background. Gldc $\mathrm{GTl}^{\mathrm{H}}$; $\mathrm{Gcsh}^{+/-}$males were generated by intercross of Gcsh ${ }^{+/-}$males with Gldc GT1/+ females. The double heterozygotes were used in timed matings 


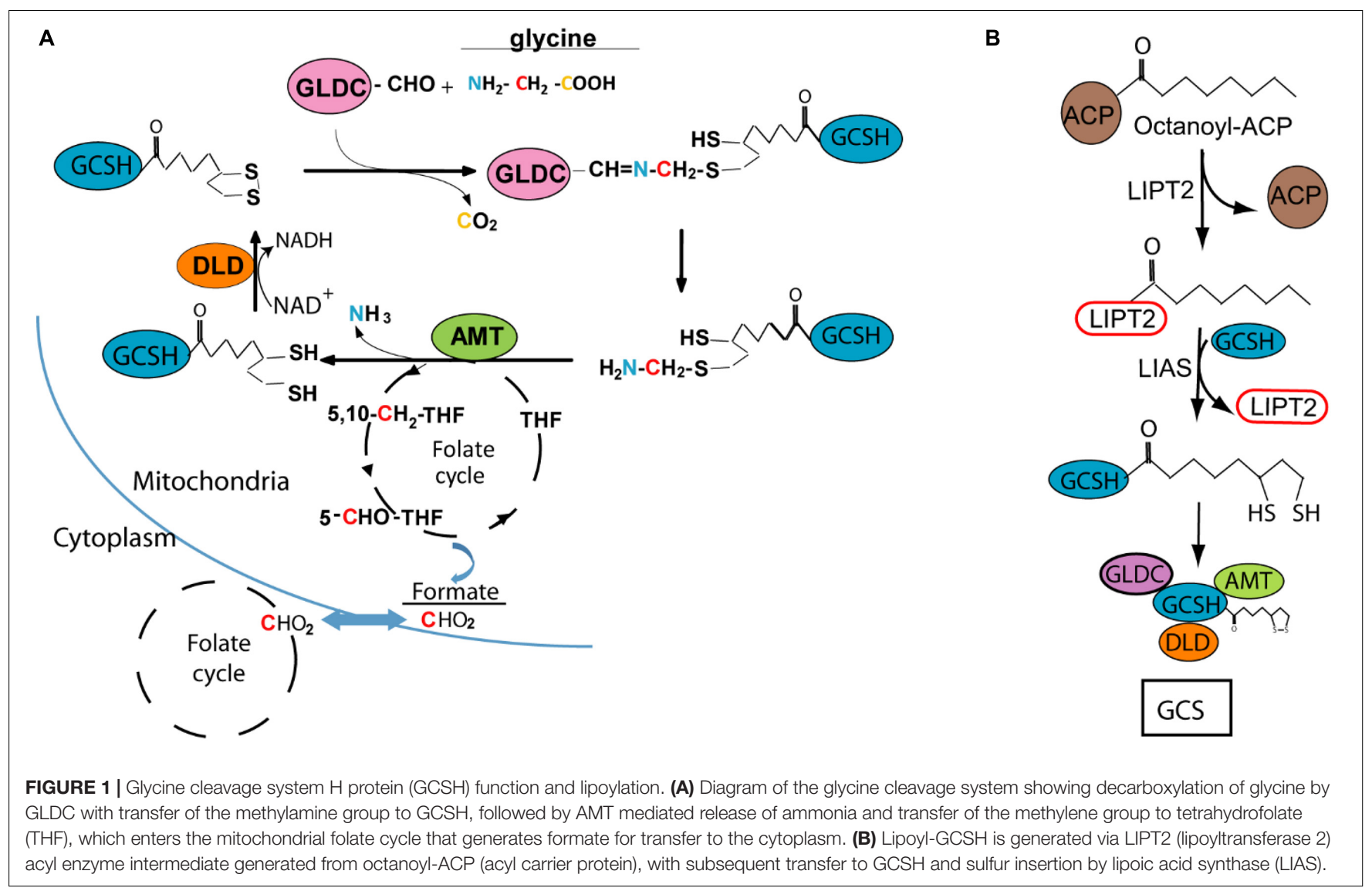

with Gldc $c^{\mathrm{GT} 1 /+}$ females in order to generate embryos of genotype, Gldc $c^{\mathrm{GT} 1 / \mathrm{GT} 1} ; \mathrm{Gcsh}^{+/-}$.

\section{Collection of Embryos and Formate Supplementation}

Timed matings were set up overnight and the day of finding a copulation plug was designated embryonic day 0.5 (E0.5). For embryo collection, the dam was killed by cervical dislocation and the uterus explanted into Dulbecco's Modified Eagles Medium (DMEM) containing 10\% fetal bovine serum, prior to dissection of embryos from the extra-embryonic membranes. In formate supplementation studies, the drinking water of pregnant dams was replaced on E0.5 with water containing $30 \mathrm{mg} / \mathrm{ml}$ sodium formate (Sigma) and mice were maintained on this water until litters were collected at E8.5 or E10.5 as in previous studies (Pai et al., 2015; Leung et al., 2017).

\section{Plasma Glycine Assay}

Blood was collected by terminal cardiac exsanguination under isofluorane anesthetic, transferred to lithium-heparin tubes (BD Microtainer), immediately centrifuged at $3,000 \mathrm{~g}$ for isolation of plasma and stored at $-20^{\circ} \mathrm{C}$. Glycine was labeled using the Kairos AccQ-tag derivation kit and analyzed on a Xevo ACQUITY UPLC I-Class PLUS System with Xevo TQ-S micro (Waters, United Kingdom). Derivatisation of glycine and UPLC-MS/MS conditions were in accordance with the manufacturer's protocol.

\section{Confocal Microscopy Imaging of E7.5 Embryos}

Confocal images of micro-dissected E7.5 DAPI-stained embryos were captured on a Zeiss Examiner LSM880 confocal microscope using a $20 \times /$ NA1.0 Plan Apochromat dipping objective. Images were also captured in reflection mode as previously described (Galea et al., 2017) and visualized as maximum projections or as $3 \mathrm{D}$ reconstructions in Fiji (Schindelin et al., 2012). For 3D reconstructions, reflection signal was enhanced using the local contrast enhancement tool (CLAHE) in-built in Fiji as previously described (Galea et al., 2018).

\section{RESULTS}

\section{The Deleted Gcsh Allele Results in Embryonic Lethality}

The Gcsh gene consists of 5 exons which generate a 1,192 nucleotide transcript encoding a polypeptide of 173 amino acids that is processed to give a mature protein of 125 amino acids. We analyzed mice carrying a 643 base pair deletion that encompassed the entire exon 3 of Gcsh, creating a frame-shift after exon 2 (amino acid 73) and a predicted null allele (here denoted $G c s h^{-}$). Gcsh ${ }^{+/-}$mice were viable and fertile and were indistinguishable from wild-type littermates. It has been reported that the electrocardiogram shows an abnormally long RR interval 
(time between $\mathrm{R}$ waves) in heterozygotes ( $n=8$ female, 7 male) compared with wild-type controls ${ }^{1}$.

No Gcsh homozygous mutant pups were recovered among the offspring of heterozygous matings, resulting in a clear distortion of the expected Mendelian ratio $\left(n=18 \mathrm{Gcsh}^{+/+}, 41 \mathrm{Gcsh}^{+/-}\right.$ and $\left.0 \mathrm{Gcsh}^{-/-}\right)$. This was consistent with data subsequently reported from the MRC Harwell preweaning lethality screen and indicates lethality of homozygous Gcsh null mice at neonatal or pre-natal stage.

Loss of function of the GCS components Gldc and Amt also results in lethal phenotypes, but null embryos survive to late-fetal stages and exhibit neural tube defects (NTDs) with varying penetrance (Narisawa et al., 2012; Pai et al., 2015; Leung et al., 2017). Therefore, we asked whether $G c s h^{-/-}$embryos were detected at pre-natal stages and if so whether NTDs were present. Experimental litters were generated by timed matings of $G c s h^{+/-}$heterozygotes and litters collected at mid-gestation. Among an initial three litters collected at E10.5, a total of twenty embryos included only two conceptuses (10\%) that genotyped as $\mathrm{Gcsh}^{-/-}$(Table 1A). Moreover, in both cases the embryonic material consisted of only a small tissue fragment within the yolk sac without typical morphology of littermates. Hence, embryonic lethality occurs prior to E10.5, as further supported by the high rate of resorption in these litters which likely corresponds to $\mathrm{Gcsh}^{-/-}$implantations (Table 1A).

Additional litters were collected at E8.5 (Table 1A) and among five litters ( $n=48$ embryos), seven implantations (15\%) contained tissue masses within the yolk sac that genotyped as $\mathrm{Gcsh}^{-/-}$, but which had not developed recognizable embryonic features such as head-folds or somites (Figure 2). Small size and abnormal embryonic structures, presumably corresponding to homozygous null embryos, was also noted by E7.5 (Figure 2).

\section{Heterozygous Gcsh+/- Mice Do Not Exhibit Elevated Plasma Glycine}

A hallmark of loss of function of the GCS in humans and mice is the presence of elevated glycine concentration in tissue and body fluids. Although this could not be assessed in homozygous mutants, we compared plasma glycine concentration in wild-type and $G c s h^{+/-}$adult mice ( $n=3$ per genotype). The plasma glycine concentration in $G c s h^{+/-}(391 \pm 72 \mu \mathrm{M}$; mean $\pm S D)$ did not significantly differ from that in wild-type mice $(347 \pm 16 \mu \mathrm{M})$, suggesting that loss of one Gcsh allele did not cause appreciable suppression of glycine cleavage system activity.

\section{Early Lethality of Gcsh Null Embryos Cannot Be Rescued by Formate Supplementation}

In addition to regulation of glycine abundance, a key activity of the GCS is the provision of glycine-derived one carbon units to folate one-carbon metabolism (FOCM), with downstream flux into nucleotide biosynthesis and methylation reactions. Suppression of FOCM is implicated in the causation of structural malformations in Gldc-deficient embryos, including NTDs and

${ }^{1}$ www.mousephenotype.org
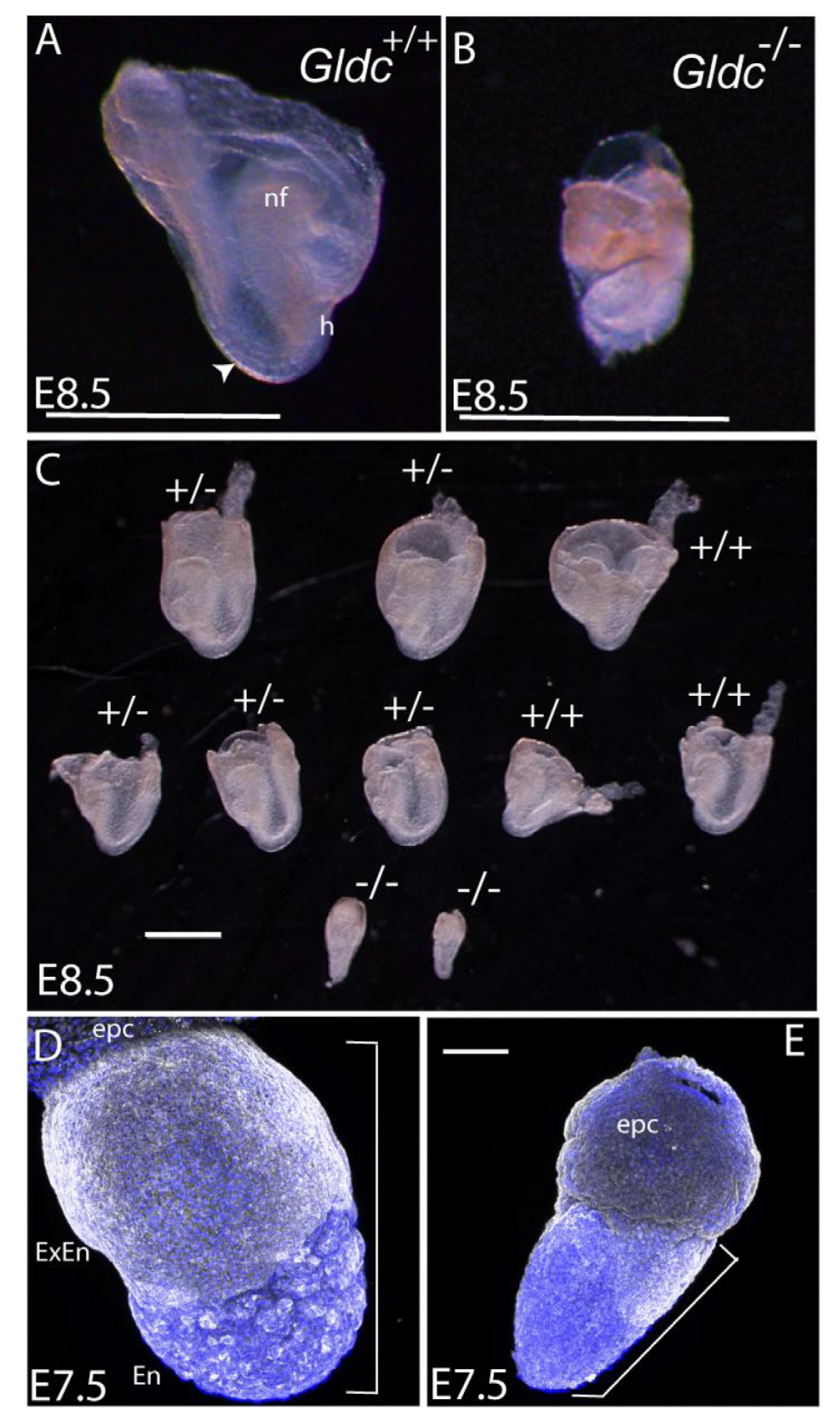

FIGURE 2 | Morphological appearance of Gcsh null embryos. (A,B) Images at 8.5 show the typical appearance of (A) a wild-type embryo (inside the yolk sac), with visible somites (arrowhead), cranial neural folds (nf), and heart loop (h), whereas (B) a typical Gcsh ${ }^{-/}$- littermate is smaller and under-developed. (C) A representative litter of embryos (contained within the yolk sac) at E8.5, in which wild-type and G/dc ${ }^{+/-}$embryos had developed to the 4-5 somite stage, with clearly visible neural folds and heart structure. In contrast, G/dc ${ }^{-}$ littermates were much smaller, with no detectable somites or age-appropriate morphological structures. (D,E) A subset of embryos were already significantly smaller than littermates at E7.5 (example in E); for comparison the region encompassing extra-embryonic/embryonic tissue is indicated by brackets (epc, ectoplacental cone; extra-embryonic endoderm; En, embryonic endoderm). Scale bar represents $1 \mathrm{~mm}(\mathbf{A}-\mathbf{C})$ or $0.1 \mathrm{~mm}(\mathbf{D}, \mathbf{E})$.

ventriculomegaly (Leung et al., 2017; Santos et al., 2020). Hence, these abnormalities can be rescued by provision of additional one-carbon groups to the folate cycle, for example via maternal supplementation with formate (Pai et al., 2015; Leung et al., 2017; Santos et al., 2020).

In order to test whether formate treatment would enhance development of $\mathrm{Gcsh}^{-/-}$embryos, heterozygous timed 
TABLE 1 | Frequency of embryo genotypes among litters from Gcsh / - intercrosses.

\begin{tabular}{|c|c|c|c|c|c|c|c|c|}
\hline \multicolumn{9}{|c|}{ A. Gcsh ${ }^{+/-} \times G_{c s h}+/-$ litters } \\
\hline & \multirow[t]{2}{*}{ Litters } & \multirow[t]{2}{*}{ Embryos (n) } & \multirow[t]{2}{*}{ Resorption (n) } & \multirow[t]{2}{*}{ Implantations litter } & \multirow[t]{2}{*}{ "Embryos" litter } & \multicolumn{3}{|c|}{ Genotype } \\
\hline & & & & & & $\operatorname{Gcsh}^{+/+}$ & $\operatorname{Gcsh}^{+/-}$ & $\operatorname{Gcsh}^{-1-}$ \\
\hline \multirow[t]{2}{*}{ E8.5 } & 5 & 48 & 1 & 9.8 & 9.6 & 18 & 23 & $7^{\star}$ \\
\hline & & & & & & $38 \%$ & $48 \%$ & $15 \%$ \\
\hline \multirow[t]{2}{*}{ E10.5 } & 3 & 20 & 7 & 9.0 & 6.7 & 6 & 12 & $2^{\star}$ \\
\hline & & & & & & $30 \%$ & $60 \%$ & $10 \%$ \\
\hline \multicolumn{9}{|c|}{ B. Gcsh ${ }^{+/-} \times G_{c s h}+/-$ litters with formate supplementation } \\
\hline & \multirow[t]{2}{*}{ Litters } & \multirow[t]{2}{*}{ Embryos (n) } & \multirow[t]{2}{*}{ Resorption (n) } & \multirow[t]{2}{*}{ Implantations litter } & \multirow[t]{2}{*}{ "Embryos" litter } & \multicolumn{3}{|c|}{ Genotype } \\
\hline & & & & & & $\operatorname{Gcsh}^{+/+}$ & $\operatorname{Gcsh}^{+/-}$ & $\operatorname{Gcsh}^{-1-}$ \\
\hline \multirow[t]{2}{*}{ E8.5-9.5 } & 4 & 36 & 1 & 9.3 & 9.6 & 10 & 19 & $7^{\#}$ \\
\hline & & & & & & $28 \%$ & $53 \%$ & $19 \%$ \\
\hline \multirow[t]{2}{*}{ E10.5 } & 4 & 20 & 7 & 6.8 & 6.7 & 6 & 11 & $3^{\#}$ \\
\hline & & & & & & $30 \%$ & $55 \%$ & $15 \%$ \\
\hline
\end{tabular}

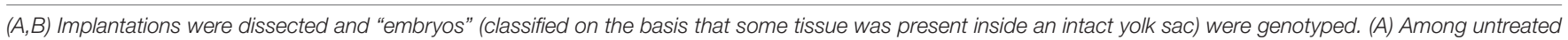

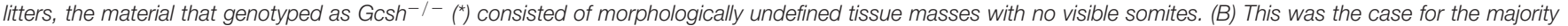

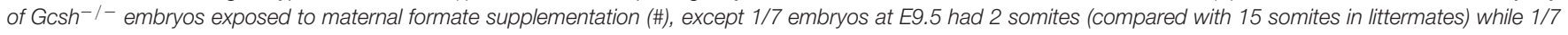

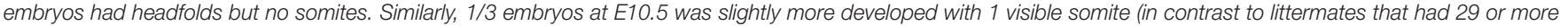
somites).

matings were performed and pregnant $G c s h^{+/-}$females were supplemented with $30 \mathrm{mg} / \mathrm{ml}$ formate in the drinking water from E0.5 as in previous studies (Pai et al., 2015). Among litters collected at E8.5-10.5, the frequency of resorption was similar to that observed among non-supplemented embryos suggesting that lethality had not been prevented (Table 1B). The majority of $\mathrm{Gcsh}^{-/-}$embryos among formate-supplemented litters resembled untreated mutants, being considerably smaller than littermates with apparent cessation of morphological development. However, a few $G c s h^{-/}$embryos (3 of 10) showed progression to a slightly later stage, with evidence of head-folds and segmentation of 1-2 somites. Nevertheless, at E9.5 these embryos exhibited developmental retardation of least 24-30 h (resembling wild-type embryos at E8.0 or younger) and showed no further developmental progression at E10.5 (Table 1B).

\section{Heterozygosity for Gcsh ${ }^{+/-}$Did Not Increase the Frequency of NTDs Among Gldc-Deficient Embryos}

Analysis of loss of function models of Gldc in mice shows that frequency of NTDs correlates with the degree of suppression of Gldc expression, defects occurring among around 25\% of Gldc ${ }^{\mathrm{GT} 1 / \mathrm{GT} 1}$ embryos and $60 \%$ of $\mathrm{Gldc}^{-/-}$embryos, which exhibit $90 \%$ and $100 \%$ reduction in Gldc mRNA abundance, respectively (Pai et al., 2015; Leung et al., 2017). The frequency of NTDs is also modified by genetic interaction with a null allele of $M t h f r$ (Leung et al., 2017). We therefore tested the hypothesis that the presence of a Gcsh null allele may have an additive effect with Gldc-deficiency leading to exacerbation of NTDs in Gldc $c^{\mathrm{GT} 1 / \mathrm{GT} 1}$ embryos.
The frequency of NTDs was assessed at E10.5 or 13.5 among litters generated by intercross of $\mathrm{Gsch}^{+/-}$; Gldc $\mathrm{GT1}^{\mathrm{GT}+}$ males and Gldc GT1/+ females (Table 2). Surprisingly, we observed NTDs among $67 \%$ ( $n=8$ of 12 ) of Gldc ${ }^{\mathrm{GT} 1 / \mathrm{GT} 1}$ embryos, which is higher than previously observed with this allele of Gldc (Pai et al., 2015). It is possible that the penetrance is influenced by alteration of the genetic background, Gcsh being on a C57BL/6N background compared with the C57BL/6J background of the Gldc GT1 line. Nevertheless, the finding that NTDs were present among only $50 \%$ of $\mathrm{Gcsh}^{+/-}$; Gldc $\mathrm{GT1}^{\mathrm{GT} 1}$ embryos compared with $83 \%$ of $\mathrm{Gcsh}^{+/+}$; Gldc ${ }^{\mathrm{GT} 1 / \mathrm{GT} 1}$ embryos (non-significant difference between genotypes) suggests that the introduction of the Gcsh null allele did not exacerbate the NTDs in Gldc GT1/GT1 embryos (Table 2).

\section{DISCUSSION}

Loss of function of Gcsh in mice results in early lethality, prior to E8.5, such that a requirement for Gcsh function in neural tube closure or later brain development and function could not be evaluated. This phenotype of Gcsh null embryos differed from that of Amt and Gldc in which embryos survive to at least perinatal stages, with death at or shortly after birth resulting from NTDs in affected pups (Narisawa et al., 2012; Leung et al., 2017). Overall, these findings indicate that the lethal effect of Gsch mutation at pre-neurulation stages is unlikely to result solely from loss of function of the GCS. However, a few formate supplemented Gcsh null embryos appeared to show a slight improvement in development compared with untreated embryos, suggesting that the suppression of FOCM may contribute in part to early lethality. The prevention of NTDs in Gldc mutant 
TABLE 2 | Frequency of genotypes and NTDs among litters from Gcsh ${ }^{+/-}$; Gldc GT1/GT1 $\times$Gcsh $^{+/+}$; Gldc ${ }^{G T 1 / G T 1}$ intercrosses.

\begin{tabular}{|c|c|c|c|c|c|c|c|c|}
\hline & Litters & $\begin{array}{l}\text { Genotype } \\
\text { Embryos }\end{array}$ & \multicolumn{3}{|c|}{$\mathrm{Gcsh}^{+/+}$} & \multicolumn{3}{|c|}{$\mathrm{Gcsh}^{+/-}$} \\
\hline E10.5 & 3 & 16 & $1(0)$ & $5(0)$ & $4(4)$ & $2(0)$ & $2(0)$ & $2(1)$ \\
\hline E13.5 & 3 & 20 & $1(0)$ & $5(0)$ & $2(1)$ & $1(0)$ & $7(0)$ & $4(2)$ \\
\hline
\end{tabular}

NTDs only arose among Gldc GT1/GT1 embryos and there was no significant effect of Gcsh genotype (Fisher Exact test).

A

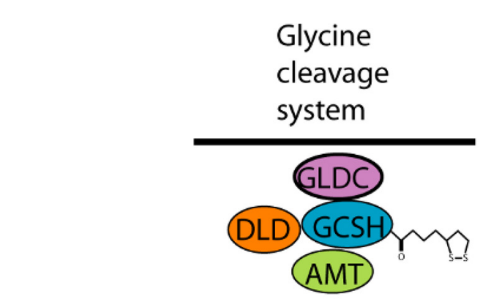

Lipoyl-enzyme

Human/mouse
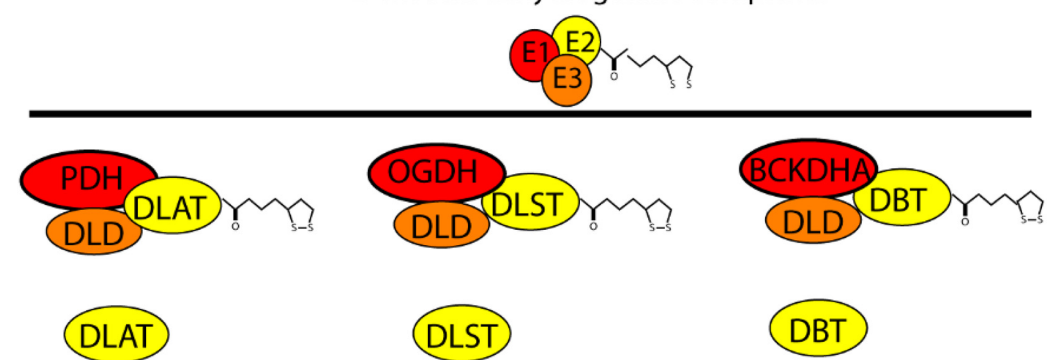

DBT

\section{B. subtilis}

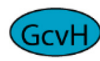

ODP2
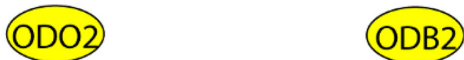

B

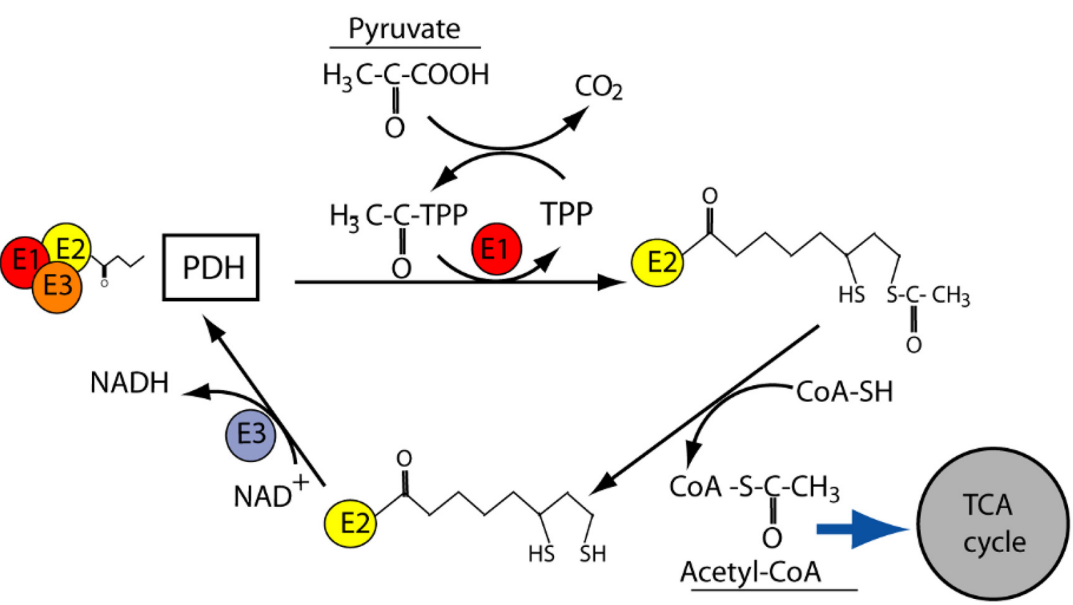

FIGURE 3 | Potential role of GCSH as a donor of lipoic acid to multiple lipoylated-proteins. (A) Lipoylation is required for function of GCSH in the glycine cleavage system, and for transfer or lipoyl groups to subunits of 2-oxoacid dehydrogenase complexes including pyruvate dehydrogenase (PDH), 2-oxyglutarate dehydrogenase (OGDH) and branched-chain alpha keto-dehydrogenase (BCKDHA). These complexes are highly conserved (orthologs in B. subtilis are shown).

(B) Possible mechanism by which GSCH acts as lipoyl-donor to the E2 subunit of pyruvate dehydrogenase, whose action leads to production of acetyl-CoA. This activity has been demonstrated in B. subtilis but not yet in human or mouse.

embryos is associated with increase in cell proliferation, perhaps via enhanced nucleotide biosynthesis, so it is possible that such an effect also supports earlier development in Gcsh mutants.

The requirement for GCSH function during early development may explain why homozygous or compound heterozygous mutations have not been reported in $\mathrm{NKH}$ patients. Similarly, NKH patients have not been found to carry $D L D$ mutations, while knockout of $D l d$ in mice results in embryonic lethality prior to E9.5, with developmental delay apparent by E7.5 (Johnson et al., 1997). This early lethality of Dld null embryos is thought to be due to the requirement for DLD function for multiple enzymes, rather than specifically the GCS. DLD acts as the so called E3 component (as dihydrolipoamide dehydrogenase) of the GCS and three other 
mitochondrial multienzyme complexes, known as 2-oxoacid dehydrogenase complexes (Figure 3A). These complexes, pyruvate dehydrogenase, $\alpha$-ketogutarate dehydrogenase and branched-chain $\alpha$-ketoacid dehydrogenase, are required for aerobic metabolism and branched-chain fatty acid synthesis. In these complexes, DLD or E3 is tightly bound to the E1 and E2 subunits where E1 is a thiamine pyrophosphate-dependent 2-oxoacid dehydrogenase. Interestingly, E2 subunits of the complexes are highly conserved and like GCSH they require covalent attachment of lipoic acid, as a cofactor, to form the active lipoyl-enzyme (Figure 3A).

Like DLD, GCSH may have functions in addition to the requirement in the GCS. This concept is supported by recent studies of the GCSH ortholog, GcvH, in B. subtilis (Cao et al., 2018a). Unlike in other bacteria, such as E. coli, where GcvH functions only in the GCS, B. subtilis GcvH can act as lipoylmoiety donor in the biosynthetic modification of other lipoic acid requiring 2-oxoacid dehydrogenase proteins. Moreover, human GCSH can substitute for loss of GcvH in B. subtilis (Cao et al., 2018a). GSCH may act as a master donor of the lipoyl group to E2 subunit of other 2-oxoacid dehydrogenase complexes such as pyruvate dehydrogenase where its function is crucial for the tricarboxylic acid (TCA) cycle (Figure 3B).

Interestingly, the transcriptional regulation of $\mathrm{GcvH}$ is also separate from other GCS components in B. subtilis, unlike in E. coli where it is co-regulated with $G c v P$ and $G c v T$ in response to glycine (Cao et al., 2018a). Tissue expression of GCSH in higher vertebrates may also occur in sites that do not express the specific GCS components (GLDC and AMT), suggesting a possible requirement beyond that in the GCS. For example, the expression level of GCSH in chick correlated with that of GLDC in liver, kidney, and brain but mRNA was also detected in other sites including heart, spleen and skeletal muscle where GLDC was absent (Kure et al., 1991). In mouse embryos Gldc is abundantly expressed throughout the neuroepithelium during neural tube closure (Pai et al., 2015) but becomes more restricted at later stages, with post-natal expression being highest in liver, kidney and brain, whereas Gcsh also is expressed in Gldc-negative tissue such as skeletal muscle (Gene expression database (GXD) ${ }^{2}$, October 2020). Similarly, human mRNA and protein databases reveal a more widespread tissue distribution of GCSH than GLDC, which is primarily expressed in liver, kidney and brain (Human Protein Atlas) ${ }^{3}$ (Uhlen et al., 2015).

\footnotetext{
${ }^{2}$ http://www.informatics.jax.org

${ }^{3} \mathrm{http}: / /$ www.proteinatlas.org
}

\section{CONCLUSION}

In conclusion, Gcsh activity is required for embryonic survival in mice. We hypothesize that Gcsh has GCS-independent activity as

\section{REFERENCES}

Autuori, M. C., Pai, Y. J., Stuckey, D. J., Savery, D., Marconi, A. M., Massa, V., et al. (2017). Use of high frequency ultrasound to study the prenatal development of a lipoate relay that is required for post-implantation development in mice and humans.

\section{DATA AVAILABILITY STATEMENT}

The original contributions presented in the study are included in the article/supplementary material, further inquiries can be directed to the corresponding author/s.

\section{ETHICS STATEMENT}

The animal study was reviewed and approved by the University College London Animal Welfare Review Board.

\section{AUTHOR CONTRIBUTIONS}

NG, K-YL, and AC conceived the study. NG, K-YL, SDC, and GG carried out the experimental work. NG and K-YL drafted the manuscript. All authors analyzed the data and edited the manuscript.

\section{FUNDING}

This work was funded by Medical Research Council (N003713) and Action Medical Research (GN2403). Research was supported by Joseph's Goal, the UCL Biological Mass Spectrometry Centre and the National Institute for Health Research Biomedical Research Centre at Great Ormond Street Hospital for Children NHS Foundation Trust and University College London.

\section{ACKNOWLEDGMENTS}

We are grateful to Chloe Santos and Diana Gold-Diaz for technical assistance and helpful discussion. The C57BL/6NTac-

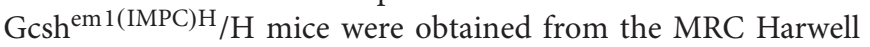
Institute which distributes these mice on behalf of the European Mouse Mutant Archive (www.infrafrontier.eu). The MRC Harwell Institute is also a member of the International Mouse Phenotyping Consortium (IMPC) and has received funding from the Medical Research Council for generating and/or phenotyping the C57BL/6NTac-Gcsh ${ }^{\text {em } 1(\text { IMPC) }} / \mathrm{H}$ mice. The research reported in this publication is solely the responsibility of the authors and does not necessarily represent the official views of the Medical Research Council. Funding and associated primary phenotypic information may be found at www.mousephenotype.org.

cranial neural tube defects and hydrocephalus in Gldc-deficient mice. Prenat. Diag. 37, 273-281. doi: 10.1002/pd.5004

Baker, P. R. II, Friederich, M. W., Swanson, M. A., Shaikh, T., Bhattacharya, K., and Scharer, G. H. (2014). Variant non ketotic hyperglycinemia is caused by 
mutations in LIAS. BOLA3 and the novel gene GLRX5. Brain 137, 366-379. doi: 10.1093/brain/awt328

Cao, X., Hong, Y., Zhu, L., Hu, Y., and Cronan, J. E. (2018a). Development and retention of a primordial moonlighting pathway of protein modification in the absence of selection presents a puzzle. Proc. Natl. Acad. Sci. US A. 115, 647-655. doi: 10.1073/pnas.1718653115

Cao, X., Koch, T., Steffens, L., Finkensieper, J., Zigann, R., Cronan, J. E., et al. (2018b). Lipoate-binding proteins and specific lipoate-protein ligases in microbial sulfur oxidation reveal an atpyical role for an old cofactor. eLife 7:e37439. doi: 10.7554/eLife.37439

Christensen, Q. H., Martin, N., Mansilla, M. C., de Mendoza, D., and Cronan, J. E. (2011). A novel amidotransferase required for lipoic acid cofactor assembly in Bacillus subtilis. Mol. Microbiol. 80, 350-363. doi: 10.1111/j.1365-2958.2011. 07598.x

Coughlin, C. R., Swanson, M. A., Kronquist, K., Acquaviva, C., Hutchin, T., Rodriguez-Pombo, P., et al. (2017). The genetic basis of classic nonketotic hyperglycinemia due to mutations in GLDC and AMT. Genet. Med. 19, 104111. doi: 10.1038 /gim. 2016.74

Cronan, J. E. (2020). Progress in the enzymology of the mitochondrial diseases of lipoic acid requiring enzymes. Front. Genet. 11:510. doi: 10.3389/fgene.2020. 00510

Galea, G. L., Cho, Y. J., Galea, G., Mole, M. A., Rolo, A., Savery, D., et al. (2017). Biomechanical coupling facilitates spinal neural tube closure in mouse embryos. Proc. Natl. Acad. Sci. U S A. 114, E5177-E5186.

Galea, G. L., Nychyk, O., Mole, M. A., Moulding, D., Savery, D., Nikolopoulou, E., et al. (2018). Vangl2 disruption alters the biomechanics of late spinal neurulation leading to spina bifida in mouse embryos. Dis. Model. Mech. 11:dmm032219. doi: 10.1242/dmm.032219

Greene, N. D., and Copp, A. J. (2014). Neural tube defects. Annu. Rev. Neurosci. 37, 221-242.

Hennermann, J. B., Berger, J. M., Grieben, U., Scharer, G., and Van Hove, J. L. (2012). Prediction of long-term outcome in glycine encephalopathy: a clinical survey. J. Inherit. Metab. Dis. 35, 253-261. doi: 10.1007/s10545-011-9398-1

Hiraga, K., Kochi, H., Hayasaka, K., Kikuchi, G., and Nyhan, W. L. (1981). Defective glycine cleavage system in nonketotic hyperglycinemia. occurrence of a less active glycine decarboxylase and an abnormal aminomethyl carrier protein. J. Clin. Invest. 68, 525-534. doi: 10.1172/jci110284

Hoover-Fong, J. E., Shah, S., Van Hove, J. L., Applegarth, D., Toone, J., and Hamosh, A. (2004). Natural history of nonketotic hyperglycinemia in 65 patients. Neurology 63, 1847-1853. doi: 10.1212/01.wnl.0000144270.83080.29

Johnson, M. T., Yang, H. S., Magnuson, T., and Patel, M. S. (1997). Targeted disruption of the murine dihydrolipoamide dehydrogenase gene (Dld) results in perigastrulation lethality. Proc. Natl. Acad. Sci. U S A. 94, 14512-14517. doi: 10.1073/pnas.94.26.14512

Kanno, J., Hutchin, T., Kamada, F., Narisawa, A., Aoki, Y., Matsubara, Y., et al. (2007). Genomic deletion within GLDC is a major cause of non-ketotic hyperglycinaemia. J. Med. Genet. 44:e69. doi: 10.1136/jmg.2006.043448

Kikuchi, G., Motokawa, Y., Yoshida, T., and Hiraga, K. (2008). Glycine cleavage system: reaction mechanism, physiological significance, and hyperglycinemia. Proc. Jpn. Acad. Ser. B Phys. Biol. Sci. 84, 246-263. doi: 10.2183/pjab.84.246

Kure, S., Kato, K., Dinopoulos, A., Gail, C., DeGrauw, T. J., Christodoulou, J., et al. (2006). Comprehensive mutation analysis of GLDC. AMT, and GCSH in nonketotic hyperglycinemia. Hum. Mutat. 27, 343-352. doi: 10.1002/humu. 20293

Kure, S., Kojima, K., Ichinohe, A., Maeda, T., Kalmanchey, R., Fekete, G., et al. (2002). Heterozygous GLDC and GCSH gene mutations in transient neonatal hyperglycinemia. Ann. Neurol. 52, 643-646.
Kure, S., Koyata, H., Kume, A., Ishiguro, Y., and Hiraga, K. (1991). The glycine cleavage system. The coupled expression of the glycine decarboxylase gene and the H-protein gene in the chicken. J. Biol. Chem. 266, 3330-3334. doi: 10.1016/s0021-9258(18)49992-9

Leung, K. Y., De Castro, S. C. P., Santos, C., Savery, D., Prunty, H., Gold-Diaz, D., et al. (2020). Regulation of glycine metabolism by the glycine cleavage system and conjugation pathway in mouse models of non-ketotic hyperglycinemia. J. Inherit. Metab. Dis. 43, 1186-1198. doi: 10.1002/jimd.12295

Leung, K. Y., Pai, Y. J., Chen, Q., Santos, C., Calvani, E., Sudiwala, S., et al. (2017). Partitioning of one-carbon units in folate and methionine metabolism is essential for neural tube closure. Cell Rep. 21, 1795-1808. doi: 10.1016/j.celrep. 2017.10.072

Mianne, J., Codner, G. F., Caulder, A., Fell, R., Hutchison, M., King, R., et al. (2017). Analysing the outcome of CRISPR-aided genome editing in embryos: screening, genotyping and quality control. Methods 12, 68-76. doi: 10.1016/j.ymeth.2017. 03.016

Narisawa, A., Komatsuzaki, S., Kikuchi, A., Niihori, T., Aoki, Y., Fujiwara, K., et al. (2012). Mutations in genes encoding the glycine cleavage system predispose to neural tube defects in mice and humans. Hum. Mol. Genet. 21, 1496-1503. doi: $10.1093 / \mathrm{hmg} / \mathrm{ddr} 585$

Oliver, D. J., Neuburger, M., Bourguignon, J., and Douce, R. (1990). Interaction between the component enzymes of the glycine decarboxylase multienzyme complex. Plant Physiol. 94, 833-839. doi: 10.1104/pp.94.2.833

Pai, Y. J., Leung, K. Y., Savery, D., Hutchin, T., Prunty, H., Heales, S., et al. (2015). Glycine decarboxylase deficiency causes neural tube defects and features of non-ketotic hyperglycinemia in mice. Nat. Commun. 6:6388.

Santos, C., Pai, Y. J., Mahmood, M. R., Leung, K. Y., Savery, D., Waddington, S. N., et al. (2020). Impaired folate 1-carbon metabolism causes formate-preventable hydrocephalus in glycine decarboxylase-deficient mice. J. Clin. Invest. 130, 1446-1452. doi: $10.1172 /$ jci132360

Schindelin, J., Arganda-Carreras, I., Frise, E., Kaynig, V., Longair, M., Pietzsch, T., et al. (2012). Fiji: an open-source platform for biological-image analysis. Nat. Methods 9, 676-682. doi: 10.1038/nmeth.2019

Shah, R. H., Northrup, H., Hixson, J. E., Morrison, A. C., and Au, K. S. (2016). Genetic association of the glycine cleavage system genes and myelomeningocele. Birth Defects Res. A Clin. Mol. Teratol. 106, 847-853. doi: 10.1002/bdra.23552

Swanson, M. A., Coughlin, C. R. Jr., Scharer, G. H., Szerlong, H. J., Bjoraker, K. J., et al. (2015). Biochemical and molecular predictors for prognosis in nonketotic hyperglycinemia. Ann. Neurol. 78, 606-618.

Uhlen, M., Fagerberg, L., Hallstrom, B. M., Lindskog, C., Oksvold, P., Mardinoglu, A., et al. (2015). Proteomics. tissue-based map of the human proteome. Science 347:1260419.

Van Hove, J. L., Kishnani, P. S., Demaerel, P., Kahler, S. G., Miller, C., Jaeken, J., et al. (2000). Acute hydrocephalus in nonketotic hyperglycemia. Neurology 54, 754-756. doi: 10.1212/wnl.54.3.754

Conflict of Interest: The authors declare that the research was conducted in the absence of any commercial or financial relationships that could be construed as a potential conflict of interest.

Copyright (c) 2021 Leung, De Castro, Galea, Copp and Greene. This is an open-access article distributed under the terms of the Creative Commons Attribution License (CC BY). The use, distribution or reproduction in other forums is permitted, provided the original author(s) and the copyright owner(s) are credited and that the original publication in this journal is cited, in accordance with accepted academic practice. No use, distribution or reproduction is permitted which does not comply with these terms. 